\title{
How can we prepare for this autumn and winter?
}

\section{Jong-Koo Lee ${ }^{\mathbb{1}}$}

Department of Family Medicine, Seoul National University College of Medicine, Seoul, Korea

Received: October 12, 2021

Accepted: October 18, 2021

Corresponding author:

Jong-Koo Lee

Department of Family

Medicine, Seoul National

University College of Medicine,

103 Daehak-ro, Jongno-gu,

Seoul 03080, Korea

E-mail: docmohw@snu.ac.kr
In the first week of October 2021, the fourth wave of the coronavirus disease 2019 (COVID-19) pandemic in the Republic of Korea continued, with a daily average of 1,806.4 cases, although the number of confirmed cases of COVID-19 has recently begun to subside [1]. In this situation, the Korean government is preparing to switch the public health emergency preparedness and response plan to "living with coronavirus," which means having a nonrestricted, safe, and responsible society in daily life, despite the current lack of a consensus. In order to achieve this goal, given the considerable diversity of opinions regarding the proper steps to take, guidance will be developed by the Covid-19 Normal Life Recovery Committee under the Prime Minister's Office, which consists of 4 divisions handling livelihood and the economy, education and culture, internal affairs and safety, and public health and medical care. However, in terms of the relationship between civil liberties, economic loss, the stringency of public health measures, and deaths from COVID-19, the Republic of Korea has achieved very good results relative to other Organization for Economic Co-operation and Development countries even without a lockdown policy. The increasing vaccine uptake rate has been accompanied by decreases in the incidence of severe COVID-19, the hospitalization rate, and the death rate, and plans are being made to lift social distancing requirements for small businesses and events. However, unless the virus fully disappears through natural selection, there will be no way to completely return to the pre-COVID-19 daily lifestyle in a strict sense.

First, we must ask: what are the prerequisites for implementing these mitigation strategies? Increasing the vaccination rate is key. A report from a German institute showed that the death rate and the number of patients will decrease significantly when the COVID-19 vaccination rate reaches $90 \%$ of those over 60 and $80 \%$ to $85 \%$ of those aged 18 to 59 [2]. This is not an easy goal to achieve because approximately 5.6 million adults who are eligible for vaccination still refuse to be vaccinated for various reasons, and vaccination is not recommended for 5.2 million children under the age of 12 , leaving about $20 \%$ of the population unvaccinated. According to recent data, although the protection provided by the Pfizer vaccine against COVID-19 infection wanes considerably-with an estimated effectiveness of roughly 20\% for preventing infection by 6 months after vaccination-the efficacy of the vaccine for preventing severe cases and hospitalizations is preserved [3]. Despite the waning of vaccine efficacy and the occurrence of breakthrough infections, the vaccine can increase the speed of recovery, meaning that vaccine uptake should continue to be promoted. The Korea Disease Control and Prevention Agency recently reported that 
unvaccinated individuals accounted for $89.8 \%$ of cases in patients over the age of 18 [4]. First of all, the vaccine uptake rate should be raised to $80 \%$ or higher through voluntary participation rather than legal regulations and coercion. Furthermore, mask use (which has increased by 95\% compared to pre-pandemic levels) in public places, subways, and buses; hand-washing; ventilation; refraining from holding or attending events; and social distancing [5] are behavioral changes that should be maintained through voluntary responsibility until the end of the pandemic.

A second question is whether relaxation of these mitigation policies will lead to an increase in the number of confirmed cases. On September 25, 2021, the number of new confirmed cases exceeded 3,000 in a single day, but the daily case load is decreasing again due to the strengthening of social distancing measures. However, the proportion of patients managed within the quarantine network is gradually falling. It is now down to $29.9 \%$, whereas just 3 or 4 weeks ago, that percentage was around $35 \%$. This means that ability of the quarantine network to conduct follow-up of confirmed cases has reached its limit [4]. Therefore, as the number of contact tracing-testing-isolation-quarantine (TTIQ) cases and the workload of health workers are increasing without recruiting new personnel, it is necessary to use an application APP that can reverse-check whether a person has overlapped with a confirmed patient so that people exposed to risk can receive prompt testing. The number of confirmed cases should decrease, thereby reducing the burden on the medical care system.

As a third question, if the vaccine uptake rate rises, will we be able to return to our daily lives? It is not an easy question to answer, due to the possibility of emerging new variants and waning vaccine efficacy; however, for the time being, freedom day such as in the UK can not be suggested. Singapore lifted many social distancing measures at the end of June 2021. However, as the number of patients has increased recently, people are again severely restricting their activities, as follows: gatherings of up to 5 people have recently been reduced to 2 , all workplaces are telecommuting and schools have switched to distance learning, and booster vaccinations have been started. However, if social distancing is strengthened again, as was the case before June, small business owners will also suffer considerably. Therefore, Singapore created a disaster relief fund worth $\$ 650$ million (about 569.8 billion Korean won) to support small business owners [6]. In contrast, in the United Kingdom (UK), the number of patients is increasing to a level similar to that observed during the second wave of COVID-19, but the hospitalization rate and death rate are 1 to 2 per 100,000 people by age group. The UK has implemented the following measures: building defenses through pharmaceutical interventions, identifying and isolating positive cases to limit transmission, supporting the National Health Service and social care, advising people on how to protect themselves and others, and pursuing an international approach [7].

In summary, how can we prepare for the fourth wave in the Republic of Korea? Even if the number of patients increases, high vaccine coverage will enable our medical care system to cope well with a surge in cases, making it unlikely that deaths will occur without medical intervention if booster vaccinations are given to high-risk groups. Although we had issues last year with the seasonal influenza vaccination, it will be given safely this year. While the United States and the UK, where outbreaks occurred in nursing homes or residential facilities, those facilities were closed and patients and residents were sent home and managed through home care, the Republic of Korea had also several outbreaks in nursing facilities in the early days of the pandemic, but the paralysis of medical institutions was prevented by managing mild cases in residential treatment facilities. In 2021, we will strengthen home care if a large surge occurs, exceeding 3,000 cases per day and continuing for more than several days. In addition, TTIQ plays a major role in interrupting transmission and reducing the number of patients, antiviral drug will support the home care policy if it can be supplied. Furthermore, opening the border requires global cooperation with many countries throughout the world, and vaccine coverage is an important indicator for permitting travel and opening the border. The Korean government will actively contribute to scaling up vaccination production and take steps to ensure the fair distribution and donation of vaccines to control this pandemic.

\section{Notes}

\section{Ethics Approval}

Not applicable.

\section{Conflicts of Interest}

The author has no conflicts of interest to declare.

\section{Funding}

None.

\section{References}

1. Korea Disease Control and Prevention Agency (KDCA). October 9th press release [Internet]. Cheongju: KDCA; 2021 [cited 2021 Oct 12]. Available from: https://kdca.go.kr/board/board.es?mid = a20501010 0008bid $=00158$ list_no $=7172318 c$ g_code $=$ \&act $=$ view\&nPage $=2$. Korean.

2. Robert Koch Institute. Epidemiologische bulletin 27/2021 [Internet]. 
Berlin: Robert Koch Institute; 2021 [cited 2021 Oct 12]. Available from: https://www.rki.de/DE/Content/Infekt/EpidBull/Archiv/2021/ Ausgaben/27_21.html.German.

3. Chemaitelly H, Tang P, Hasan MR, et al. Waning of BNT162b2 vaccine protection against SARS-CoV-2 infection in Qatar. N Engl J Med 2021 Oct 6 [Epub]. https://doi.org/10.1056/NEJMoa2114114.

4. Korea Disease Control and Prevention Agency (KDCA). September 22 press release [Internet]. Cheongju: KDCA; 2021 [cited 2021 Oct 12]. Available from: https://kdca.go.kr/board/board.es?mid = a20501010 0008bid $=00158$ list_no $=7170298 c g \_c o d e=$ sact $=$ view8nPage $=5$. Korean.
5. Cho KS. Changes in infectious diseases, health behaviors, and medical uses during COVID-19 pandemic in the Republic of Korea, 2020. Public Health Wkly Rep 2021;39:2750-64. Korean.

6. Tan A. Dining in capped at 2, WFH the default: what you need to know about Covid-19 rules from Sept 27 [Internet]. The Straits Times; 2021 Sep 24 [cited 2021 Sep 28]. Available from: https://www.straitstimes. com/singapore/health/dining-in-capped-at-two-wfh-the-defaultamong-6-key-changes-as-spore-tightens-covid.

7. HM Government. Covid-19 responses: autumn and winter plan 2021 [Internet]. London: HM Government; 2021 [cited 2021 Oct 12]. Available from: https://www.gov.uk/government/publications/covid19-response-autumn-and-winter-plan-2021. 